\title{
Le roi, le forgeron et les premiers hommes dans l'ancienne société Kongo
}

The king, the smith and the first people in ancient Kongo

Luc de Heusch

\section{OpenEdition}

1 Journals

Édition électronique

URL : http://journals.openedition.org/span/251

DOI : 10.4000/span.251

ISSN : 2268-1558

Éditeur

École pratique des hautes études. Sciences humaines

\section{Édition imprimée}

Date de publication : 1 septembre 1975

Pagination : 165-179

ISSN : 0294-7080

\section{Référence électronique}

Luc de Heusch, «Le roi, le forgeron et les premiers hommes dans l'ancienne société Kongo »,

Systèmes de pensée en Afrique noire [En ligne], 1 | 1975, mis en ligne le 09 juillet 2013, consulté le 14 novembre 2019. URL : http://journals.openedition.org/span/251 ; DOI : 10.4000/span.251 
LE ROI, LE FORGERON ET LES PREMIERS HOMMES

DANS L'ANCIENNE SOCIETE KONGO

par Luc de HEUSCH

PROPOS - Cette note fait partie d'une recherche d'ensemble sur la pensée symbolique des sociétés de lanque et de culture konoo (Zaĩre, Angola, République du Conoo). De nombreuses études leur ont été consacrées depuis un demi-siècle. Les chroniques anciennes relatives à $1^{\prime}$ 'ancien royaume de Konqo, fondé vraisemblablement au 15e siècle, ont également été exploitées.

Nous montrons ici, que les rites d'investiture des chefs mpangu au début du siècle éclairent certains aspects de l'intronisation royale decrite au 17 e siecle à condition d'examiner soigneusement les traditions mythiques relatives à la première humanité. Une première prise de contact avec le terrain a eu lieu chez les Mata du Bas-zaĩre au cours d'une mission organisée par l'Institut National des Musées Zairrois durant 1'êté 1974 .

Plusieurs traditions mythiques konoo font état d'un premier âge de l'humanité, marqué par le passage d'une créature étrange douée de pouvoirs magiques extraordinaires = Nzondo. Ce héros culturel qui n'avait qu'une jambe, un pied, un oeil, laissa aux hommes un mystérieux alaive sans manche (nsengele mbele), symbole de l'unité, de la puissance et du savoir du peuple kongo. Mais après la mort de Nzondo, les hommes ne surent plus se servir de cet instrument magique; ils finirent par le perdre (1). Ou encore, ils se disputèrent à son sujet (2). Quoi qu'il en soit, les tribus kongo commencèrent alors à se disperser : les émigrants s'installèrent sur les terres de petits hommes mystérieux, les mbaka, que l'on identifie tantôt à des nains, tantôt à des pyg̣mées. La tradition mpangu en fait de grands forgerons (3). Le peuple de Nzondo est composé de créatures humaines incomplètes, alors que les mbaka sont des hommes de taille réduite; les mythes substituent aisément 1 'une à I'autre ces figures symboliques equivalentes. Ce qui retiendra notre attention ici, c'est leur association directe ou allusive

(1) FU KIAU, Le Mukongo et le monde qui l'entourait, Kinshasa, 1969 , p. 116.

(2) R. WANNYN, L'art ancien du métal au Bas-Congo, Champles (Belgịque), 1961, p. 23. J. CUVELIER, "Traditions conọolaises", Congo, II, 4, 1930, p. 470.

(3) J. VAN WING, Etudes Bakongo, I, Histoire et socioloaie, Bruxelles, 1921, p. 79. 
au travail du fer, au prestige et à la puissance qu'il confère.

Les Mpangu, dont le nom même évoque celui d'une ancienne province du royaume de Kongo, n'ont cessé, jusqu'à une êpoque récente, de confier au forgeron le soin d'investir les "chefs couronnës", qui ont le droit de porter le bonnet mpu à l'instar du souverain et des dignitaires de la cour, à l'époque où celle-ci était florissante. Après l'effondrement du royaume, au cours des XVIIIe et XIXe siècles, ces chefs couronnés tentèrent apparemment de restaurer à échelle réduite, et dans un cadre purement clanique, le pouvoir magico-religieux issu de Kongo dia Ntotiza, le Kongo du roi. Ils étaient de plus en plus rares, lorsque le père Mertens fit son enquête en pays mpangu dans les années qui précèdent la seconde guerre mondiale. Cet observateur est donc $l^{\prime}$ un des derniers témoins d'une tradition d'autant plus intéressante que les forgerons façonnaient à cette occasion les bracelets de fer nlunga, que l'on peut considérer comme le symbole le plus éminent de l'ancienne royauté sacrée. En effet, une chronique manuscrite de 1624 , nous apprend que le souverain portait au bras droit cet insigne vénérable qui était censé représenter le royaume entier. Le témoin portugais décrit le nzunga (ma zomgo) comme "un bracelet de fer doré"; il précise que le pays était appelé "Congo de fer" (1).

Les chefs "couronnés" mpangu possédaient aussi au XXe siècle un glaive appelé mbele nsanga, qui n'est pas sans évoquer 1 'arme magique du poutoir originel détenu par le mythique. Nzonào : à ceci près que celui-ci était. dépourvu de manche, alors que Mertens fait état d'une lame' munie d'un manche orné de cuivre jaune (2). Voici posé le problème des relations rituelles du pouvoir et de ia forge. Les traditions rapportées par Mertens

(1) Le témoin oculaire est un chanoine portugais de Sao Salvador qui assista en 1622 au sacre de Pedro II et à celui de son fils Garcia I en 1624 (cité par L. JADIN, "Aperçu de la situation du Congo et rite: d'élection des rois en 1775 , d'après le P. Cherubino da Savona, missionnaire au Congo de 1759 à 1774", BulZ. de Z'Inst. Hist. Belge de Rome, fasc. XXXV, 1962 , p. 404.).

(2) J. MERTENS, Les chefs couronnés chez les Bakongo orientaux (Etude de régime successoral), Bruxelles, 1942, p.77. 
montrent clairement que le forgeron légitime l'autorité traditionnelle, en tant que maitre de l'initiation. C'est lui qui ouvre la corbeille sacrée et en extrait l'un des bracelets ancestraux qu'il passe au bras du futur chef. C'est lui qui forgera pendant la retraite initiatique du candidat-chef,les bracelets nouveaux que celui-ci ajoutera à celui que ses prédécesseurs lui ont aìnsi directement légué (Mertens, p. 71). A ce titre, le forgeron est appelé nganga nzunga, le maitre du bracelet de fer, (idem, p. 53). C'est lui encore qui prélève sur le cadavre du chef investi, au moyen du mbele nsanga précisément, des poils d'aisselle, des rognures d'ongle, un morceau de peau en divers endroits; ces reliques sont enfermées dans une corne d'antilope nsyesye enveloppée d'une étoffe rouge. La corbeille est composée des reliques de tous les chefs couronnés, ainsi que de celles qui ont été prélevées sur le corps de la femme qui fut rituellement investie en même temps que chacun d'eux, la ndona. On $y$ ajoute les bracelets, enduits d'huile et de poudre rouge nkula, ceux de la main gauche recouvrant ceux de la main droite ( $i$ dem, p. 22).

L'antilope nsyesye attire immediatement l'attention. Non identifiée par Mertens, elle est certainement l'antilope naine nsiese (Guevei coerulus, Hamilton Smith) à qui les Kongo attribuent une intelligence hors pair. Elle apparait sous le vocable tsese dans un conte recueilli par Bittremieux : grâce à un goupillon magique, elle provoque l'apparition de la foudre et de la pluie (1). Chez les Mpangu, la couleur roug̣e, associée à la corbeille des ancêtres, renvoie au même code saisonnier : lorsque l'orage approche, les hommes éteignent les feux et éloignent de la case tout ce qui est rouge pour que Nzazi, la foudre, les épargne (2). Or l'ouverture solennelle de la corbeille des ancêtres par le forgeron se déroule, comme le rite protecteur précédent, dans le plus grand silence (Mertens, p. 27). Il s'exécute sur la place publique tandis que l'on bat deux instru-

(1) L. Bittremieux, "Le Palmier Divin", Revue Congolaise, 4, 1913, p. 45 à 51 .

(2) J. VAN WING, Etudes Bakongo, II, Religion et Magie, Bruxelles, 1938, p. 79 . 
ments de fer façonnés grossièrement à l'image des marteaux de forge dont ils portent le nom $(n z u n d u)$. Il faut mettre en parallèle cette description avec un passage de la chronique royale que je viens d'évoquer : au cours du couronnement du roi Garcia I en 1624, "trois hommes portent chacun deux instruments de fer de la grandeur de petites esoopettes de bonne facture et, de temps en temps, ils les frappent et produisent un son semblable à celui que fait un forgeron quand il frappe avec le marteau sur l'enclume. Ils appellent cela zimie, c'estl'insigne royal le plus ancien et seul le roi peut l'employer et le duc de Batta" (I). Je reviendrai sur la signification de l'intervention rituelle du duc de Batta (ou Mbata), qui régnait sur 1 'une des provinces les plus importantes du royaume.

Lorsque guidé par le forgeron, le chef mpangu moderne suivi de ses proches, entre dans la maison initiatique, il meurt symboliquement. A la fin du troisième jour, le forcoron revient avec un silure qu'il introduit dans la bouche de chacun des participants (Mertens, p. 54). Ce rite est rigoureusement semblable à celui auquel sont soumis les jeunes gen's et les jeunes filles qui, après avoir subi la "mort-nkita", sont identifiés à des esprits de l'eau à la fin de la grande initiation religieuse kimpasi, dont Van wing a recueilli les derniers vestiges (2). Pendant la durée de la retraite initiatique, le candidat-chef ne peut porter de pagne noir ou foncé, mais seulement un vêtement blanc (Mertens, p. 54). Cette couleur rituelle est celle des esprits aquatiques dans I'ensemble du monde kongo. Le jour de son entrée, le candidat se débarrasse des bracelets provisoires (nlunga $i$ nyanga) composés des mêmes herbes de brousse (nyanga) que celles qui ont servi à la confection de la maison initiatique. Or cette "herbe à couvrir" (nom quénérique de tout chaume) (3) intervient dans le rituel kimpasi

(1) JADIN, , op.cit., p. 401.

(2) J. VAN WING, op. cit. chap. VI.

(3) P. SWARTENBROECKX, Dictionnaire kikongo et kituba-françis, Bandundu, 1973, p. 427. 
lors de la pêche au silure qui s'effectue dans une fosse en forme de croix : aux deux extrémités latérales de celle-ci, "on plante une petite botte d'herbes nianga entourée de cendres de bois" (1). Cette indication rapide montre suffisamment que les mêmes éléments symboliques interviennent dans l'initiation religieuse et l'initiation au pouvoir politique. On notera en particulier que le candidat-chef s'enduit, pour se fortifier, des cendres du foyer qui brûle à l'intéxieur de la clôture rituelle. Introduit, comme les initiés du kimpasi, dans le monde de la brousse qui est en propre celui des esprits, le candidat-chef est intégré par le forgeron dans l'univers historique et culturel : après s'être débarrassé des bracelets d'herbe (2), le futur chef porte des bracelets de fer qu'il s'exerce à faire sonner, tandis que le forgeron l'initie à la généalogie de ses ancêtres (Mertens, pp. 55-56). L'aarément de ceux-ci se manifeste par une chasse fructueuse comportant obligatoirement la capture de trois espèces d'antilopes (idem,p. 58).

Enfin, le forgeron fait sortir rituellement le candidat de la maison initiatique en le tirant par le petit doigt : à trois reprises celui-ci résiste, présentant seulement la tête dans l'embrasure de la porte (p. 59). La scène fiqure manifestement un accouchement, d'autant plus que la case-matrice est un endroit clos, muni d'une ouverture unique et si réduite qu'il faut ramper pour y pénétrer (p. 52); ainsi extirpé de sa retraite, le candidat est lavé comme un nouveau-né (p. 60). L'initiateur le fait monter sur la pierre de forge et lui passe au bras gauche les nouveaux bracelets qu'il a confectionnés. Le nouveau chef renoue avec le passé historique en énumérant toutes les rivières que ses ancêtres ont traversées depuis le départ de Kongo dia Ntotila, le kongo du roi. Le rôle du forgeron n'est pas terminé : il trace sur le sable, avec le pied, un dessin cruciforme sur lequel il dépose une croix de bois de palme (mbasa). La cérémonie se termine par un épisode bien curieux : le nouveau

(1) J. VAN WING, op. cit., p. 216 .

(2) On notera que la corbeille initiale du chef de Mpangu ne contenait que des "anneaux de fines herbes" (VAN WING, Etudes Bakongo, I, P. 781 
chef disparaft en forêt et un homme nu (qếnéralement un esclave) dont une cuisse est peinte en blanc, l'autre en noir, se lance à sa recherche. Lorsqu'il l'aperçoit, le premier agite ses bracelets en signe de jole. L'émissaire entraine alors la foule. Transporte en palanquin au village en compagnie de sa ndona, le chef se met à danser en agitant son sabre mbele nsanga, tout en faisant sonner ses bracelets. Le forgeron intervient enfin une dernière fois : il enduit le couple d'un mélange d'huile de palme, de poudre rouge $n k u z a$ et de sang de bouc pour le rendre invulnérable (pp. 63-66).

I1 ressort de cette description que la sacralité du pouvoir politique est conférée par le forgeron, maitre du bracelet. Le symbolisme de cet objet mériterait évidemment d'être approfondi; trois bracelets sont particulièrement importants dans la mesure où ils représentent les trois premières lignées du clan (p. 69). Cette division tripartite du clan est un véritable archétype chez les Kongo. Mais il est plus intéressant encore d'apprendre que les Mpangu articulent parfois à cette notion sociologique une conception évidement mythique : le premier et le second bracelet portent le nom des jumeaux,Nsimba et Nzuzi (p. 61).

Ces informations, certes incomplètes, me paraissent susceptibles d'éclairer l'aspect le plus remarquable de l'ancienne royauté sacrée, à laquelle toutes ces traditions se réfèrent explicitement : les chroniques anciennes montrent clairement que le roi est intronisé par un grand prêtre, le Mani Vunda, dont il dépend rituellement. Celui-ci ne cessera de conduire les rites d'investiture traditionnels après la christianisation du royaume. Le nom même de vunda dérive d'un personnage historico-mythique, Nsaku ne Vunda, un prêtre autochtone qui guérit le conquérant Lukeni (ou Ntinu) des convulsions dont il souffrait avant qu'il ne fondât la capitale (1). Or, il se fait que le clan.nsaku auquel appartient le premier Mani Vunda connu, est censé descendre d'ancêtres nains ou pygmées, mbaka, originaires du sud de la région de Loanda (2). Cette tradition doit

(1) J. CUVEIIER, L'ancien royaume de Congo, Bruxelles, 1946, p. 15 .

(2) J. CUVELIER, idem. 
Etre évidemment interpretée en termes mythiques puisque de nos jours encore, les Mpangu attribuent a des autochtones nains, aujourd'hui disparus, des talents de forgerons que les Pygmeses authentiques n'ont évidemment jamais possédés (1). En revanche, 11 y a lieu de croire que le travail du fer etait pratiqué au sud du fleuve, bien avant l'arrivé des conquérants kongo, en rupture de ban avec le petit royaume vungu dont ils étaient originaires, au nord du fleuve. En effet, une datation au carbone 14 concernant le nord-est de l'Angola, permet de situer l'âge du fer dans cette région au premier siècle de notre ère (2). Il est donc probable d'une très ancienne et puissante corporation de forgerons exerçait son autorite politique et religieuse sur les agriculteurs autochtones avant même 1'arrivée des fondateurs de la dynastie kongo au XVe siècle. Je me range ici à l'avis de Randlès qui estime qu'"il n'est guère vraisemblable que ce fut, comme certains l'ont prétendu, Lukéni et la conquête bakongo qui introduisit le fer au sud du fleuve" (3).

Examinons le problème de plus près. Wannyn qui a étudié l'art ancien du métal au Bas-Congo écrit que les réponses à des centaines d'interrogations sont concordantes : "le premier ntotela (roi) a êté 'maitre de forges' sinon forgeron" (4). Mais ses informateurs ajoutent aussitôt que le travail du fer ne fut jamais réservé au roi ou à quelques dignitaires': tous les hommes libres avaient accès à la profession, au prix d'une initiation. Ces mêmes informateurs insistent en revanche sur

(1) VAN WING, Etudes Bakongo, I, p. 79

(2) J.D. CLARK, Prehistoric Cultures of North-East Angola and their significance in Tropical Africa, Lisbonne, 1963. Cet auteur indique (p. 18) la date suivante : $1880 \pm 80 \mathrm{BP}=$ $70 \mathrm{AD}$, selon l'estimation UCLA 170. Mais Fergusson, G.J. et Libby, W.F. (UCLA radio carbon Dates II, Radiocarbon 5, 1963, p. 17) donnent pour cette meme analyse (UCLA 170) une date légèrement plus tardive ; $1800 \pm 80 \mathrm{BP}=150 \mathrm{AD}$. Je remercie Daniel Cahen d'avoir bien voulu me fournir cette précision.

(3) W. G.L. RANDLES, L'ancien royaume du Congo des origines à la fin duXIXe siècle, Paris,Mouton, 1968, p. 79.

(4) R. WANNYN, op. cit., p. 59. 
l'interét que le roi portait aux "nombreux ateliers de forgerons installés sur les collines proches de la capitale". Rapportant de son côté la tradition orale kongo, il y a une quarantaine d'annees, Cuvelier écrit : "Mbanza Kongo, ou du moins un quartier de la capitale, s'appelait Mbangala Nzundu Tadi (...) par allusion sans doute au Ngangula Ne Kongo au roi forgeron" (1). Tout ceci porte à croire, on le verra, que le souverain ne devait ce titre qu'à l'initiation que lui conférait le Mani Vunda, en sa qualité de représentant des forgerons autochtones. Il faut réinterpréter soigneusement la traduction proposée par Cuvelier de la formule "Mbangala Nzundu Tadi". Nzundu désigne bien le marteau, mais tadi, qui siqnifie pierre, renvoie évidemment à l'enclume, celle-là même sur laquelle monte le chef couronné mpangu lors de son investiture. Le terme mbangala pourrait signifier effectivement "massue", mais il s'agirait alors d'une redondance peu satisfaisante. Dans I'ancienne tradition, conservée au nord du fleuve chez les Yombe, Mbangala n'est autre que le nom de la mère originelle, la femme aux neuf seins qui engendra les neuf ancêtres des clans kongo (2).

Cette devise forgeronne de la capitale m'a été récitée, sous une forme corrompue et aujourd'hui incompréhensible, par un informateur mbata, comme la devise même du royaume : "Kongo di ntadi banguna nzundu ". Par ailleurs, chez les mêmes Mbata, un prêtre du fétiche protecteur Mpungu a récité spontanément devant mol une formule proche de celle rapportée par Cuvelier en 1930 : "Bangala tadi nzundu ", pour commenter la présence d'un marteau miniature dans un coquillage spiralé (kodi) contenant d'autres objets de fer minuscules, parmi lesquels on notera la représentation du soleil et de la lune.

(1) J. CUVELIER, "Traditions congolaises", Congo, II, 4, 1930, p. 487 .

(2) L. BITTREMIEUX, Mayombsch Idioticon, deel II, Gand, 1923, p. 347 . 
Les traditions relatives' a 1 'arrivé des conquérants venus du nord du fleuve sous la conduite de l'aventurier Lukeni ou Ntinu, fondateur de la dynastie qui règne à Mbanza Kongo, font état de deux evènements.

1. Lukeni vainquit le chef autochtone Mabambolo Mani Pangalla qui régnait sur la région de sao salvador (1). On hésitera à rapprocher le terme Pangalla (Mpangala) de Mbangala car les deux mots sont distincts en kikongo : Mpangala désigne le jour du marché ou le marché (2). Après la conquête, les descendants du seigneur de Mpangala continuèrent à régner sur une fraction du territoire (3). On notera aussi qu'un haut dignitaire qui portait toujours le titre de "mani Pangala" figure au début du XVIIIe siècle parmi les quatre électeurs du comte de soyo, 1'un des vassaux du roi. Il êtait l'un des "féticheurs" les plus puissants (4).

Randles fait observer que le nom Mabambolo n'est très probablement qu'une corruption de Mbumbulu, le magicien primordial de la tradition mpangu (5). Mais ce personnage appartient evidemment au mythe. Il fut initie par un demiurge celeste, Nzala Mpanda. Celui-ci planta en terre un pilon qui devint l'arbre mbota (Milletia Deweivri). Il planta aussi un bananier qui mûrit le jour même à midi. Il traversa une rivière et laissa l'empreinte de son pied sur un rocher. Après sa mort, il ressuscita grâce à une corne d'antilope qui émit un sifflement. Il mourut une seconde fois mais son cadavre se redressa dans la tombe et il remonta au ciel (6).

De toute évidence, la fonction de "Mani Pangala" exercée par le chef autochtone Mbumbulu et ses successeurs dans le comté du soyo était de caractère religieux. On pourrait aisément

(1) W.G.L. RANDLES, L'ancien royaume du Congo des origines a la fin du XIXe siècle, Paris-La Haye, 1968, p. 19, d'après CAVAZZI, 1ib. II, 86.

(2) P. SWARTENBROECKX, op. cit., p. 352.

(3) J. CUVELIER, "Traditions congolaises", CongoII, 4, 1930,p.487.

(4) J. CUVELIER, Re Zation sur le Congo du Père Laurent de Lucques (2700-l727), Bruxelles, 1953, p. 114.

(5) W. G. L. RANDLES, op. cit. p. 20.

(6) J. VAN WING, Etudes bakongo, II, p. 164. 
démontrer que la légende de Nzala Mpanda n'est qu'une transformation du mythe d'origine concernant la première humanité, le peuple de Nzondo, puissant magicien qui possédait le glaive du pouvoir et du savoir (1). Une variante particulièrement intéressante de ce mythe, nous ramène à notre point de départ, la devise forgeronne du royaume, car elle introduit Mbangala, la mère originelle aux neuf seins : son fils â̂né Makaba nous est présenté dans ae récit comme le chef des magiciens nzondo (2). Il apparait de plus en plus clairement que la devise forgeronne du royaume est en relation directe avec divers mythes d'origine concernant le mystérieux peuple nzondo, mâtre du glaive originel. Il est intéressant d'observer que les Manyanga établissent un rapport explicite entre la disparition du glaive de Nzondo et la perte du savoir métallurgique : "L'enclume est un outil précieux. Laissé dans les mains des enfants (il) devient instrument à décortiquer les amandes" (3). La tradition orale du royaume introduit dans sa propre histoire le personnage légendaire de Makaba, doublet de Nzondo : bien qu'ainé des neuf neveux du premier souverain, il fut exclu du partage du pouvoir entre les divers chefs du clan (4). Il est remarquable qu'au cours de cet évènement mémorable, tous les chefs dansèrent avec le glaive mbele (5).

On commence à comprendre que les symboles du pouvoir associé à la forge, sont attribués par les Kongo à une première humanité mythique, avec laquelle les chefs autochtones vaincus (Mbumbulu, Nsaku ne Vunda) sont en rapport direct. Le clan dominant nsaku, qui prétend descendre des mythiques mbaka, leur impose son pouvoir religieux (6). Face à I'ainé de ce clan, Nsaku ne Vunda, de qui il reçoit l'investiture, le fondateur de la nouvelle dynastie se trouve exactement dans la même position

(1) L. BITTREMIEUX, op. cit., deel II, p. 556.

(2) L. BITTREMIEUX, op. cit., deel I, p. 347.

(3) FU KIAU, op. cit., p. 116.

(4) J. CUVELIER, "Traditions congolaises" Congo, 2, 1931,p.201.

(5) Idem, P. 200 .

(6) Il est singulier que dans la tradition recueillie par Cuvelier, (région de Makuta), les Mbaka auraient ignoré la métallurgie, alors que les informateurs de Van Wing leur attribuent les amas de scories attestant l'ancien travail de la forge (CUVELIER, "Traditions congolaises", 1930, p. 479). 
rituelle que les modemes chefs couronnes mpangu, issus de la dispersion des clans. Nous abordons ici le second évènement "historique" majeur de la conquête kongo su sud du fleuve.

2. Le conquerant Lukeni (ou Ntinu Wene) fut pris d'un accès de folie qui fut guéri à l'intervention de ssaku ne Vunda, prêtre autochtone, maître de l'agriculture, de la pluie et de la chasse. Il fixa sa résidence à quatre lieues de la "montagne de la séparation" où s'effectua le partage du royaume. Le roi s'allia alors au clan nsaku qui conserva le pouvoir sur la province qui allait devenir le "duché" de Mbata (1). La préséance rituelle du prêtre du clan nsaku sur le roi est clàirement exprimée dans le titre prestigieux qui lui sera réservé tout au long de l'histoire agitée du Congo :Vunda signifie, en effet, Alné (2). La tradition orale (région de Tumba) a conservé le souvenir du pouvoir rituel éminent $d u$ chef $d u$ clan autochtone dominant : "Celui qui n'était pas intronisé par le seigneur Nzaku n'était pas roi" (3). A la fin du XVIIIe siècle encore, le Mani Vunda a le pas sur le souverain (4). Le Mani Vunda et le duc de Mata (qui appartiennent à l'origine au même clan autochtone) sont lesdeux personnages les plus importants de la cour selon une source vaticane de la fin du XVIe siècle publiée par Cuvelier et Jadin (5). C'est le duc de Mbata qui, après avoir fait l'éloge du nouveau souverain, l'entraîne par la main vers le trône où il reçoit notamment le bracelet de fer (description de l'intronisation de Garcia I en 1624) (6). Ie rôle rituel du duc de Mata, qui agit

(1) CUVELIER, L'ancien royaume de Congo, pp. 13-15.

(2) SWARTENBROECKX, op. cit., p. 702 .

(3) J. CUVELIER, "Traditions congolaises", 1930, p. 472.

(4) L. JADIN, "Relation sur le royaume du Congo du P. Raimondo da Dicomano, missionnaire de 1791 à 1795". BulZ. des séances de l'ARSC, Bruxelles, 1957, III, 2, pp. 328-330.

(5) J. CUVELIER et L. JADIN, L'ancien Congo d'après les archives romaines, Bruxelles, 1954, p. 199.

(6) L. JADIN, "Aperçu de la situation au Congo et rite d'election des rois", op. cit., p. 401 . 
de concert avec le Mani Vunda, évoque d'autant plus l'intervention du forgeron chez les Mpangu, qu'il est le seul avec le roi a pouvoir detenir ces instruments de fer que 1 'on bat au cours du couronnement de manière à produire "un son ressemblant à celui que fait le forgeron quand il frappe avec le marteau sur l'enclume" (1). Ce rite est rigoureusement équivalent, on l'a déjà vu, à celui qui avait lieu récemment encore, lors de l'investiture des chefs couronnés mpangu.

Les deux évènements historiques que je viens de commenter, la victoire du fondateur de la dynastie sur deux chefs autochtones, se réfèrent en fin de compte à un seul et même texte mythologique : Mbumbulu et Nsaku se trouvent respectivement en rapport avec les Nzondo et les Mbaka, c'est-à-dire la première humanité détentrice du secret perdu de la forge. Il est remarquable que la tradition orale enregistrée par Cuvelier identifie parfois Nsaku, le premier Mani Vunda, avec Makaba, le chef des Nzondo, à moins qu'elle ne fasse du premier un descendant du second : c'est ce qu'exprime clairement la formule "Nsaku wa Makaba" (2). En tout état de cause, Nsaku et Makaba occupent une position d'ainesse dans des traditions homologues et inverses : le premier conserve la position de Vunda, c'est-à-dire d'aîné, face au roi; le second est, lors du partage du royaume, un aîné malheureux, dépossédé.

L'histoire de la conquête d'une terre dont les mâttres étaient très probablement associés à la forge, n'est qu'une reprise en charge du passé mythique. Il faudrait montrex encore que le héros céleste Nzala Mpanda, l'initiateur du chef autochtone Mbumbulu, n'est lui-même qu'une transformation de 1 'ancien dieu du ciel kongo, Bunzi, mastre de la pluie. Sans entrer dans les détails de cette démonstration, on notera que la résurrection de Nzala Mpanda grâce au sifflement d'une corne d'antilope, peut être mis en rapport avec le rite funéraire accompli par le forgeron mpangu qui enferme dans la corne de l'antilope naine, martresse de la pluie, les reliques prélevées sur le cadavre du

(1) L. JADIN, idem.

(2) J. CUVELIER, "Traditions congolaises", 1931, p. 200. 
chef couronné. Dans cette perspective, le forgeron kongo apparaît comme le représentant du démiurge céleste responsable du cycle saisonnier et de la fécondité. De cet ancien Dieu du ciel, dont le Dieu des missionnaires, Nzambi, a obscurci la véritable image, Nzala Mpanda n'est qu'une épiphanie; on vient de voir que celui-ci à son tour recouvre la figure de Nzondo, détenteur du glaive magique primordial et maître de la première humanité incomplète. Or Nzondo est aussi responsable, dans la tradition manyanga, des eaux diluviennes qui sont à l'origine du fleuve Zaīre (1). Après la disparition de Nzondo, les hommes perdirent le glaive mbele et se dispersèrent. On comprend mieux peut-être, à la suite de ce détour mythologique, pourquoi cette arme figure parmi les insignes du pouvoir transmis aux chefs mpangu par le forgeron, héritier rituel du peuple de Nzondo. Si les chroniqueurs anciens $n$ 'en parlent jamais lorsqu'ils évoquent les symboles du pouvoir royal, en revanche la tradition orale recueillie par Cuvelier y accorda une importance considérable (2) : le premier souverain remit le couteau, symbole de la puissance royale, à chacun de ses neveux lorsqu'ils dansèrent solennellement avant la dispersion des clans (2). Les chefs de clan mbata que j'ai rencontrés en 1974 au zaïre, non loin de la frontière de l'Angola, possèdent toujours glaives et bracelets de fer que leurs ancêtres auraient reçus jadis du roi de Kongo. Mais toute trace de l'initiation par le forgeron a disparu.

(1) FU KIAU, op. cit., p. 116 .

(2) J. CUVELIER, "Traditions congolaises", 1930, p. 482. 


\section{DISCUSSION}

A. de SURGY

L. de HEUSCH

N. ECHARD

L. de HEUSCH

$J . P . ~ C O L L E Y N$

L. de HEUSCH

A. de SURGY
- Comment explique-t-on l'association entre les hommes d'avant et la forge?

- On peut seulement supposer que l'activité des forgerons etait plus intense au sud du fleuve. Il est curieux qu'au nord du fleuve, d'ou viennent les conquérants, les auteurs ne font jamais mention du rôle rituel du forgeron, bien que le roi du Loango soit intronisé par le grand prêtre de Bunzi, maitre de la pluie, qui est l'exact équivalent du Mani vronda. La rencontre des conquérants avec les maittres autochtones de la forge a été pensée comme mythe.

- Y a-t-il une différence de statut entre les fondeurs et les forgerons proprement dits ?

- Il est impossible de répondre à cette question car le travail traditionnel de la forge a complètement disparu au cours des đernières années chez les Mbata et, semble-t-il, dans l'ensemble du monde kongo; celui-cl s'étend, ne l'oublions pas, entre Kinshasa et l'océan, c'est-à-dire dans un lieu de très ancienne colonisation.

- Peut-on supposer que le fer aurait d'abord été travaillé au sud du fleuve?

- Cela n'est pas exclu. On a longtemps supposé que la diffusion du fer parmi les Bantous s'est faite à partir de l'Afrique occidentale ou le site le plus ancien (Nok) date environ du milieu du premier millénaire avec J-C. Mais une récente fouille archéologique suggère l'existence d'un centre métallurgique plus ancien encore en Tanzanie. Les datations proposées doivent cependant êt re retenues avec prudence.

- Dans le sud du Togo la tradition rapporte que lorsque les populations traversèrent la forêt, elles y trouvèrent de petits hommes rouges ayant une queue, qui travaillaient le fer. Dans les sanctuaires des vodun, on trouve aussi la représentation d'un esprit de la chasse qui ne possède qu'un pied et un bras : la puissance est toujours impaire. 
J. ROUCH

J.P. COLLEYN

G. DIETERLEN

G. $L E$ MOAL
Y. CISSÉ

- Dans la tradition initiatique peul rapportée par Hampate $B a$, un groupe de travalileurs du bols, les Kule, joue un rôle très important. Est-ce que cette corporation existe chez les kongo comme groupe distinct des forgerons?

- Les Kule disent qu'ils sont d'origine bambara. Dans les villages minianka ou demeure un forgeron, il est interdit aux Kule de creuser un mortier : en effet, le forgeron minianka travaille le fer et le bois.

- Les Kule sont considérés comme impurs, ils sont censés pratiquer l'inceste alors que le forgeron est toujours pur. Ce qui me-frappe dans l'exposé de L. de Heusch, c'est l'extraordinaire similitude des mythes kongo et des mythes bambara. On dirait que l'on a à faire au même matériel travaillé differemment.

- En bambara on appelle forgeron ceux qui ont fabrique, soit un outillage technique en pierre, en bois ou en fer, à usage agricole, soit un outillage de fondation.

L. de HEUSCH - Très souvent en Afrique centrale bantoue, les forgerons qui ne forment jamais une caste, travaillent le bois. Chez les Pende par exemple, le forgeron est aussi sculpteur.

- Chez les Bobo également, le travail du bois est associé au. travail du fer. 